\title{
PURIFICAÇÃO DO SUBPRODUTO DO PROCESSO DE EXTRAÇÃO DE ESTEVIOSÍDEO ${ }^{1}$
}

\author{
Denise Maria Malachini MIOTTO2,*, Nádia Regina Camargo Fernandes MACHADO ${ }^{2}$
}

\section{RESUMO}

O caramelo consiste no subproduto do processo de extração dos edulcorantes das folhas de Stevia rebaudiana Bertoni. Esse subproduto apresenta teores significativos dos edulcorantes esteviosídeo e rebaudiosídeo A não extraídos no processo e grande quantidade de compostos das folhas que lhe conferem, respectivamente, sabor doce e coloração escura. Desta forma, a retirada dos compostos das folhas presentes no caramelo torna possível seu reaproveitamento como edulcorante. Portanto, o caramelo foi purificado por meio de adsorção em zeólitas modificadas, CaX e MgX. Foram realizados dois experimentos: um teste de saturação dos adsorventes para avaliar sua capacidade adsortiva e um teste de máxima clarificação para determinar a máxima purificação alcançada por adsorção em zeólitas. Os resultados mostraram que CaX é o adsorvente mais eficaz. As zeólitas podem ser reutilizadas por até duas vezes, necessitando regeneração em seguida. O teste de máxima clarificação apresentou soluções quase límpidas, com altos níveis de clarificação (80-90\% dos compostos com maior afinidade), mas elevadas retenções dos edulcorantes ( 70\%), pois as zeólitas conseguem reter grande parte dos pigmentos da solução de caramelo, arrastando também esteviosídeo e rebaudiosídeo A, com baixo rendimento de recuperação dos edulcorantes, mas considerado satisfatório tendo em vista que o caramelo, apesar de rico em edulcorantes, não tem aplicação atualmente.

Palavras-chave: esteviosídeo; rebaudiosídeo A; adsorção; zeólitas.
\end{abstract}

\section{SUMMARY}

PURIFICATION OF THE BY-PRODUCT OF THE STEVIOSIDE EXTRACTION PROCESS. Caramel is the by-product of the sweeteners extraction process of Stevia rebaudiana Bertoni leaves. This by-product shows significant contents of the sweeteners stevioside and rebaudioside A not extracted in the process and large quantity of leaf components, that give it, respectively, sweet flavor and dark color. Like this, the retreat of the leaf components of the caramel makes possible its reuse as a sweetener. Therefore, the caramel was purified by adsorption in modified zeolites, CaX and MgX. Two experiments were accomplished: a test of saturation of the adsorbents to evaluate its adsorption capacity and a test for maximum clarification to determine the maximum purification reached by adsorption in zeolites. Starting from the results, it was verified that CaX is a more effective adsorbent. Zeolites can be reused up to twice, needing regeneration soon after. The test for maximum clarification showed almost clear solutions, with high clarification levels (80-90\% of the compounds with larger likeness), but with high retentions of the sweeteners $(70 \%)$, because zeolites retain most of the pigments of the caramel solution, but they also drag stevioside and rebaudioside A, resulting in a process with low yield in relation to the recovery of the sweeteners, but considered satisfactory, in view that caramel, in spite of being rich in sweeteners, has no use at present.

Keywords: stevioside; rebaudioside A; adsorption; zeolites.

\section{1 - INTRODUÇÃO}

Nos dias atuais, o homem tem procurado uma vida mais saudável e seus hábitos alimentares vêm sendo modificados pela introdução de novos produtos químicos na sua dieta. Seja por cuidados com a estética ou problemas de saúde, o homem está substituindo o açúcar por produtos conhecidos como edulcorantes, compostos com sabor semelhante ao da sacarose, porém com baixo valor calórico ou completamente sem calorias [1].

Dentre os edulcorantes naturais, pode-se destacar o esteviosídeo e rebaudiosídeo A, principais glicosídeos diterpênicos extraídos das folhas de Stevia rebaudiana Bertoni, com grande aplicação na indústria alimentícia devido a sua estabilidade frente ao calor e a uma ampla faixa de $\mathrm{pH}$. O esteviosídeo é o glicosídeo presente em maior quantidade nas folhas de Stevia, possui dulçor

\footnotetext{
1. Recebido para publicação em 17/06/2003. Aceito para publicação em 22/12/2003 (001151).

2. Departamento de Engenharia Química, Universidade Estadual de Maringá - PR, Bloco D-90, Av Colombo, 5790, Campus Universitário, CEP: 87020-900, Zona 07, Maringá-PR-Brasil.E-mail: denisemmm@deq.uem.br. E-mail:nadia@deq.uem.br

*A quem a correspondência deve ser enviada.
}

150 a 300 vezes maior que a sacarose, mas apresenta forte sabor amargo residual. O rebaudiosídeo A, por sua vez, é o segundo maior componente edulcorante da planta e apresenta 250 a 400 vezes o dulçor da sacarose, possuindo sabor residual levemente amargo. O teor de edulcorantes total nas folhas de Stevia rebaudiana encontra-se na ordem de 10\%, em massa [2].

A Empresa Steviafarma Industrial S.A., localizada em Maringá/PR, é a única empresa brasileira a realizar o processo de extração dos edulcorantes das folhas de Stevia rebaudiana. Seu atual processo industrial gera um subproduto denominado caramelo, que consiste em um pó de coloração marrom esverdeada, com sabor amargo e aroma adocicado. O caramelo contém teores significativos de esteviosídeo e rebaudiosídeo A, não extraídos no processo, além de grande quantidade de pigmentos das folhas. Esse material, desde a implantação da fábrica, vem sendo estocado em tanques, sem qualquer utilidade [6].

Dentro desse contexto, estudou-se um processo alternativo de recuperação do caramelo. Para tanto, realizou-se a clarificação e purificação de soluções aquosas de caramelo por meio da adsorção dos compostos coloridos das folhas, que geram o sabor amargo e a coloração escura, por zeólitas modificadas, CaX e MgX [4]. 
A opção pelas zeólitas modificadas como adsorvente deu-se a partir de resultados de clarificação do extrato aquoso do chá de Stevia, por adsorção em zeólita CaX, realizada por MORAES \& FERNANDES MACHADO [7]. A extensão a MgX fundamentou-se em um processo patenteado por KOTARO \& TOKUO [3], que utilizaram adsorvente composto de alumínio e magnésio na purificação de esteviosídeo.

Zeólitas são aluminossilicatos cristalinos, cuja estrutura é composta pela combinação tridimensional de tetraedros $\mathrm{SiO}_{4}$ e $\mathrm{AlO}_{4}^{-}$, unidos entre si através de átomos de oxigênio comuns. A carga negativa formada pelo tetraedro de alumínio é compensada por cátions que, no caso dos adsorventes citados, consistem em $\mathrm{Ca}^{+2} \mathrm{e}$ $\mathrm{Mg}^{+2}$. Esses cátions neutralizam as cargas negativas da zeólita X, gerando as formas modificadas CaX e MgX [5].

\section{2 - MATERIAIS E MÉTODOS}

O caramelo em pó fornecido pela Steviafarma Industrial consiste em 40-50\% em massa de edulcorantes, além de uma infinidade de compostos das folhas não determinados devido a sua extrema complexidade.

As zeólitas CaX e MgX foram preparadas a partir da zeólita de partida NaX do tipo Baylith WE894 da Bayer, granulada, com esferas entre 3,0 e 4,0mm, por meio de troca iônica. A primeira, com solução de $\mathrm{CaCl}_{2}$ a $20 \%$ (p/p), gerou CaX, e a segunda, com solução de $\mathrm{MgCl}_{2}$ a 18\% (p/p), produziu MgX [4].

Foram realizadas bateladas de adsorção com soluções de caramelo em duas concentrações distintas: 4\% (4g caramelo/100mL água deionizada) e 7\% (7g caramelo/100mL água deionizada), onde para cada $10 \mathrm{~mL}$ de solução de caramelo foram adicionados $6 g$ de adsorvente.

A adsorção ocorreu por meio de contato entre o adsorvente e a solução de caramelo, deixada por 24 horas à temperatura ambiente, com agitação branda e controlada em banho termostático.

Os compostos das folhas que conferem coloração escura ao caramelo absorvem abundantemente a luz visivel, de modo que a Espectrofotometria UV-VIS apresentou-se eficaz na medida da clarificação da solução de caramelo. Uma varredura no espectro visível da solução de caramelo não apresentou nenhum pico intenso de absorção devido à grande mistura de compostos. Para avaliar a retenção dos compostos que absorvem a luz visivel, optou-se por realizar varreduras a fim de determinar a clarificação do adsorvato ao longo do espectro (400 a 750nm), com medida a cada $10 \mathrm{~nm}$ em Espectrofotômetro Shimadzu UV-1203.

A Equação (1) foi utilizada na determinação da clarificação no espectro visível.

\% Clarificaç ão $=\left[1-\left(\frac{\text { Absorbânci }_{\lambda} \_ \text {após }}{\text { Absorbânci } a_{\lambda \_} \text {antes }}\right)\right] * 100$

Realizaram-se dois experimentos de adsorção. O primeiro foi denominado teste de saturação pois utili- zou, por quatro bateladas consecutivas, a mesma zeólita como adsorvente, sendo adicionada em cada batelada uma nova solução-estoque de caramelo. O teste de saturação teve como objetivo verificar a eficácia das zeólitas como adsorvente na purificação do caramelo.

Em contrapartida, o segundo experimento realizou três bateladas consecutivas de adsorção com a mesma solução de caramelo, adicionando-se adsorvente novo a cada batelada. O objetivo do teste de máxima clarificação consistiu em verificar quanto era possivel purificar o caramelo através de adsorção em zeólitas. Os produtos obtidos foram submetidos à análise por cromatografia líquida de alta eficiência, realizada em Cromatógrafo Líquido Modelo CG-480C, com Coluna $\mathrm{NH}_{2}$ Brownlee $22 \mathrm{~cm}$, fase móvel acetonitrila/água 80:20 (v/v), vazão de $1,5 \mathrm{~mL} / \mathrm{min}$, temperatura ambiente e pressão de 80atm. O detector UV fez a leitura a $210 \mathrm{~nm}$. Foram determinados os teores de edulcorantes presentes no caramelo antes e após a adsorção, assim como a redução dos principais compostos das folhas detectados.

\section{3 - RESULTADOS E DISCUSSÃo}

\section{1 - Teste de saturação}

O comportamento das quatro bateladas de adsorção está demonstrado nas Figuras 1 a 4, em que nota-se a busca pela uniformidade de adsorção dos pigmentos das folhas do caramelo em toda a faixa do espectro analisado, em cada batelada de adsorção.

Observando a Figura 1, verifica-se que a curva de melhor resultado diz respeito ao comportamento da solução de caramelo 4\% frente às zeólitas CaX novas, denominadas de CAX_1DIA (primeiro dia de utilização). A partir do segundo dia, as zeólitas já se encontravam parcialmente saturadas, e o perfil da curva variou em função do equilibrio atingido em cada uma das bateladas.

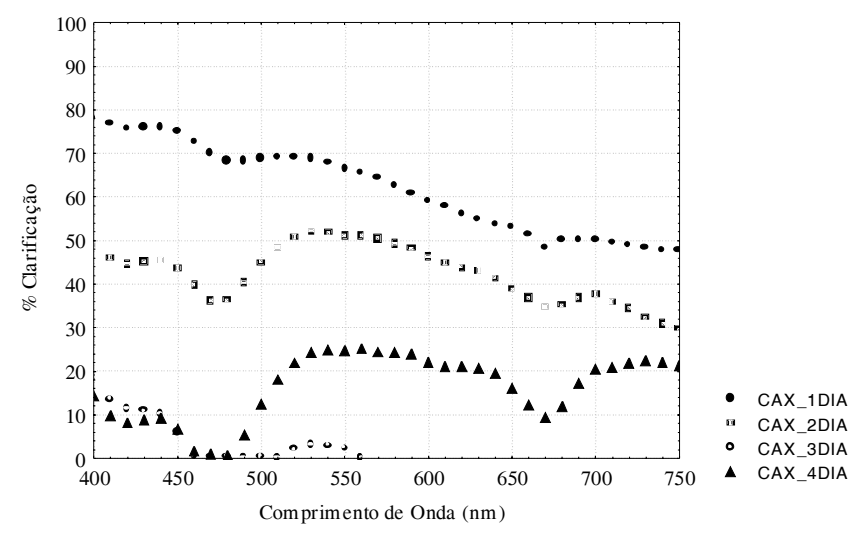

FIGURA 1. Clarificação da solução de caramelo $4 \%$ com zeólitas CaX durante 4 dias

Observa-se que de CAX_1DIA para CAX_2DIA a clarificação foi reduzida, sendo de forma mais pronunciada para menores comprimentos de onda. Isto significa que os compostos que absorvem a luz visível nestes comprimentos de onda foram preferencialmente adsor- 
vidos ao primeiro contato, fazendo com que logo no início do segundo contato estas substâncias atingissem a capacidade máxima de adsorção, sem serem muito adsorvidas. Já com as demais substâncias o equilíbrio foi atingido nos dois dias, e o pior desempenho no segundo contato se deve à redução de sítios de adsorção disponíveis.

O terceiro contato mostrou claramente a saturação da zeólita para diversos compostos, pois somente aqueles de grande afinidade ainda conseguiram ser reduzidos. A parte da curva que não aparece no gráfico resultou em valores negativos de clarificação, uma vez que a absorbância da solução após o contato com o adsorvente foi maior que a da solução-estoque. Isto pode ser explicado avaliando-se a busca pelo equilíbrio. Como as zeólitas apresentavam-se muito saturadas, ao entrarem em contato com a solução-estoque, a concentração da maioria das substâncias coloridas era maior na zeólita do que na solução, ocorrendo dessorção.

Para CAX_4DIA, verifica-se que a dessorção do terceiro dia fez com que a zeólita novamente fosse capaz de adsorver compostos da solução com o perfil assemelhando-se aos demais dias.

Em MGX_1DIA e MGX_2DIA, apresentados na Figura 2, o comportamento foi idêntico, apenas com uma previsível redução no poder adsorvente de MgX. Já na terceira e quarta bateladas, MGX_3DIA e MGX_4DIA, respectivamente, a zeólita mostrou-se completamente saturada com grande parte dos compostos dessorvidos e, ao invés de ser clarificada, a solução foi concentrada.

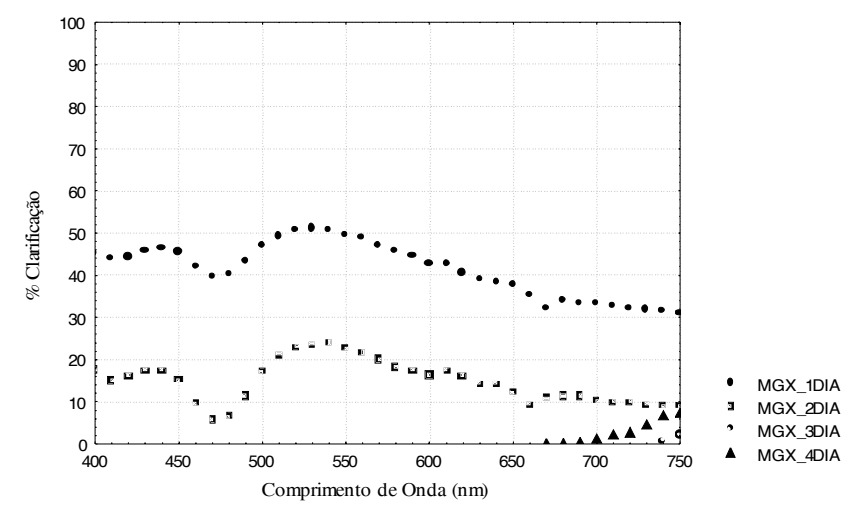

FIGURA 2. Clarificação da solução de caramelo $4 \%$ com zeólitas MgX durante 4 dias

As curvas indicadas na Figura 3 mostram variação de comportamentos da zeólita $\mathrm{CaX}$ frente à solução de caramelo $7 \%$, ao longo dos dias. No primeiro contato, os compostos com maior afinidade são adsorvidos mais facilmente, de modo que na segunda batelada, os compostos que absorvem em maiores comprimentos de onda são adsorvidos mais efetivamente. Esta mudança de comportamento é resultado, novamente, da diferenciação dos sítios de adsorção. Como as zeólitas novas adsorveram preferencialmente os compostos verdeamarelados e amarelos no primeiro contato, no segundo contato o gradiente de concentrações dos demais compostos foi maior, favorecendo sua adsorção.

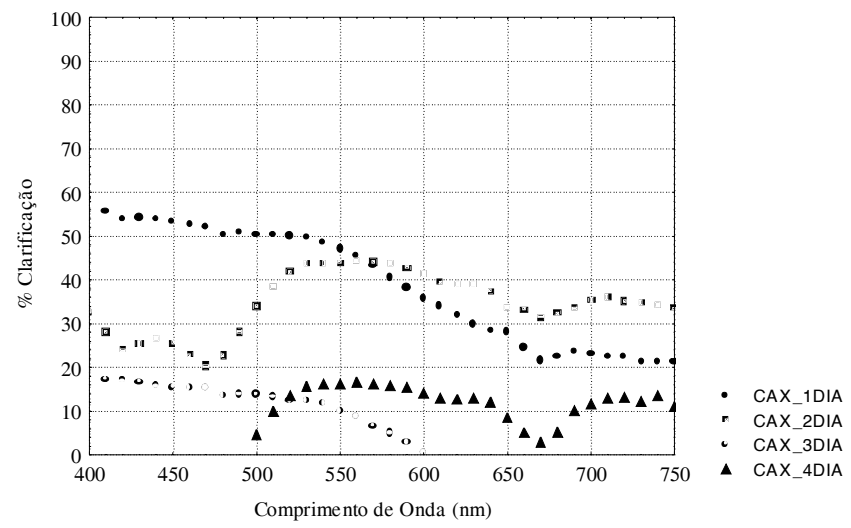

FIGURA 3. Clarificação da solução de caramelo $7 \%$ com zeólitas CaX durante 4 dias

O mesmo raciocínio pode ser estendido para CAX_3DIA e CAX_4DIA, com similar variação de comportamento, só que neste caso as clarificações, como era de se esperar, foram bem menores, chegando a valores negativos no terceiro dia, exatamente como ocorreu com a solução de caramelo 4\% frente às zeólitas CaX.

De acordo com a Figura 4, em contato com a solução de caramelo 7\% as zeólitas MgX na primeira batelada apresentaram o resultado esperado, com maior afinidade pelos compostos com coloração de vermelho-púrpura a azul. Na segunda batelada, houve uma pequena melhora na clarificação dos compostos menos adsorvidos, e redução para os demais.

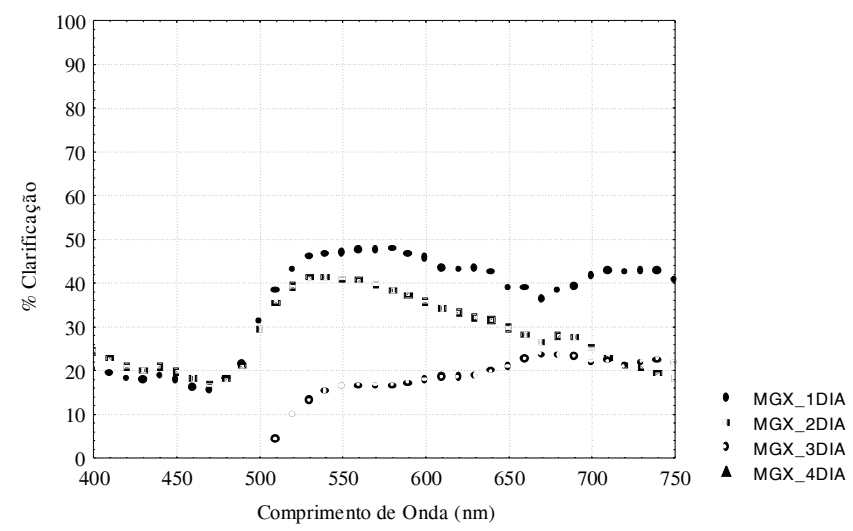

FIGURA 4. Clarificação da solução de caramelo $7 \%$ com zeólitas MgX durante 4 dias

No terceiro contato somente foram adsorvidas substâncias com maior afinidade, aquelas que absorvem a luz visivel em comprimentos de onda a partir de 500nm, e no quarto dia, a zeólita apresentava-se completamente saturada, ocorrendo apenas dessorção.

O perfil de adsorção de MgX frente à solução de caramelo $7 \%$ é similar ao perfil frente à solução de caramelo 4\%. A maior diferença reside na concentração das soluções, o que levou o adsorvente a ser um pouco mais eficiente para concentrações maiores, porque apesar de obter porcentagens de clarificação menores, a saturação ocorreu com maior tempo de contato. 


\section{2 - Teste de máxima clarificação}

Neste teste foram realizadas três bateladas, em seqüência, de clarificação da mesma solução de caramelo por adsorção em zeólitas novas. Assim, 20mL de solução de caramelo foram clarificadas com $36 \mathrm{~g}$ de zeólita. A cada dia foi determinado o perfil de clarificação para observar seu comportamento frente a soluções cada vez mais diluídas. Os resultados estão demonstrados nas Fiquras 5 e 6.

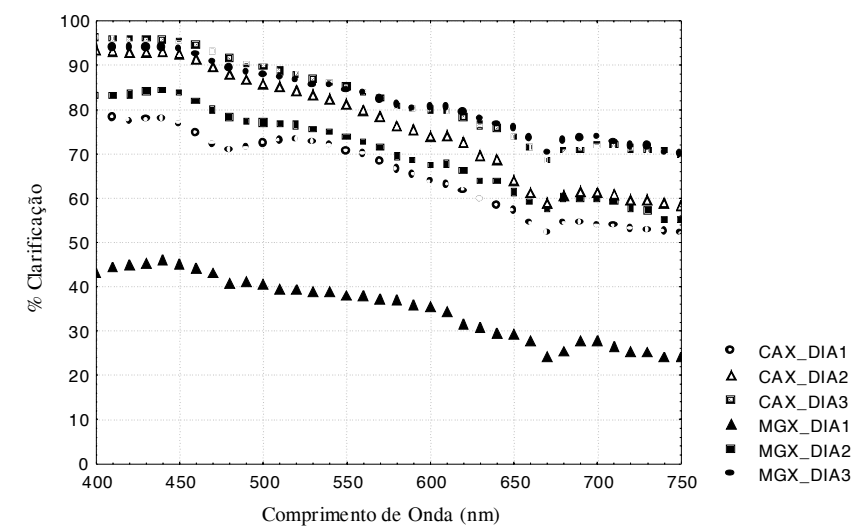

FIGURA 5. Clarificação máxima de solução de caramelo $4 \%$ com zeólitas CaX e MgX

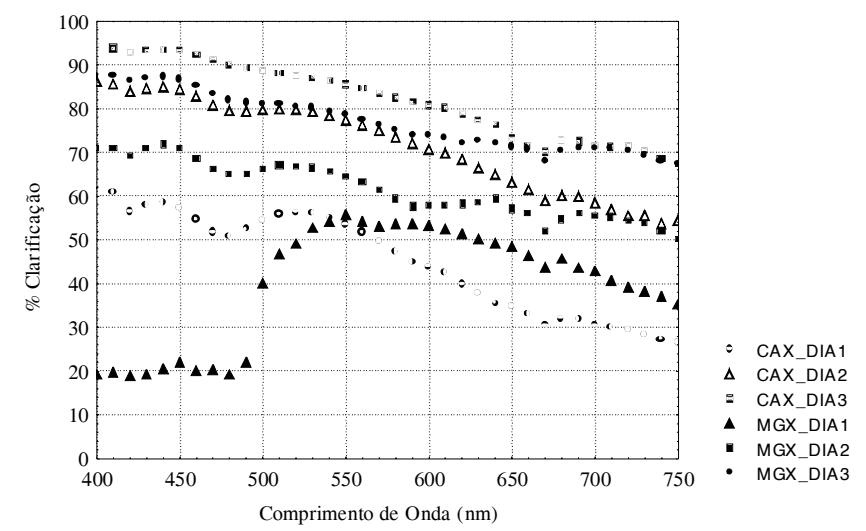

FIGURA 6. Clarificação máxima de solução de caramelo $7 \%$ com zeólitas CaX e MgX

A Figura 5 mostra o desempenho das zeólitas CaX e MgX na clarificação da solução de caramelo 4\%. As curvas CAX_DIA1, CAX_DIA2 e CAX_DIA3 se referem às clarificações da solução por CaX durante as três bateladas. Observa-se que o perfil das curvas não foi modificado e a solução atingiu elevados valores de clarificação. Os resultados das zeólitas $\mathrm{MgX}$, indicados por MGX_DIA1, MGX_DIA2 e MGX_DIA3, assim como para as zeólitas CaX, não demonstraram mudanças de comportamento frente à solução, que a cada batelada estava mais diluída.

À medida que a solução foi sendo clarificada, sua concentração foi diminuindo e o gradiente de concentração entre as espécies coloridas em solução e na zeólita foi se tornando cada vez menor, reduzindo progressivamente a taxa de adsorção, a ponto de se parar o teste no terceiro dia. É importante ressaltar que os dois adsorventes apresentaram praticamente o mesmo resultado após a terceira clarificação, sendo ambos considerados eficientes, resultando em soluções com elevado grau de purificação.

Para a solução de caramelo 7\%, mostrada na Figura 6 , os dois adsorventes começaram com comportamentos distintos, mas ao final apresentaram um perfil muito semelhante. As zeólitas CaX mantiveram seu comportamento inicial, havendo uma desaceleração gradativa na clarificação durante as bateladas. Tendo em vista que a solução torna-se cada vez menos concentrada, esse resultado foi considerado esperado.

Já para as zeólitas MgX, o perfil sofreu algumas modificações. No primeiro dia seu comportamento foi normal, com clarificações mais efetivas a partir de $500 \mathrm{~nm}$. No segundo dia a solução tinha reduzido significativamente a quantidade dos compostos de coloração vermelho-púrpura a azul e continuava com altas concentrações das demais substâncias, o que levou o adsorvente a compensar este desequilíbrio. Na terceira batelada a solução se apresentava ainda menos concentrada e a zeólita manteve seu comportamento frente a soluções diluídas. Diferentemente da solução de caramelo 4\%, neste caso as zeólitas CaX foram mais eficientes ao final dos três dias de contato.

\subsection{Cromatografia líquida de alta eficiência}

Como o objetivo do estudo foi reter, por adsorção, os compostos coloridos e recuperar os edulcorantes de interesse, realizou-se análise de cromatografia líquida de alta eficiência com as amostras clarificadas por três bateladas consecutivas, para quantificar os pigmentos e edulcorantes antes e após a clarificação. As Tabelas 1 e 2 mostram estes resultados.

TABELA 1. Resultados da cromatografia líquida para solução de caramelo $4 \%$

\begin{tabular}{cccc}
\hline Resultados Avaliados & $\begin{array}{c}\text { Caramelo 4\% } \\
\text { (solução-estoque) }\end{array}$ & $\begin{array}{c}\text { Caramelo 4\% } \\
\text { clarificado com CaX }\end{array}$ & $\begin{array}{c}\text { Caramelo 4\% } \\
\text { clarificado com MgX }\end{array}$ \\
$\begin{array}{c}\text { \% Edulcorantes } \\
\text { \% Compostos das } \\
\begin{array}{c}\text { Folhas } \\
\text { \% Redução dos }\end{array}\end{array}$ & 61,41 & 64,59 & 65,83 \\
$\begin{array}{c}\text { Edulcorantes } \\
\text { \% Redução dos }\end{array}$ & 38,59 & 35,41 & 34,17 \\
Compostos das Folhas & $\# \# \# \#$ \#\#\# & 69,28 & 76,13 \\
\hline
\end{tabular}

TABELA 2. Resultados da cromatografia líquida para solução de caramelo $7 \%$

\begin{tabular}{cccc}
\hline Resultados Avaliados & $\begin{array}{c}\text { Caramelo 7\% } \\
\text { (solução-estoque) }\end{array}$ & $\begin{array}{c}\text { Caramelo 7\% } \\
\text { clarificado com CaX }\end{array}$ & $\begin{array}{c}\text { Caramelo 7\% } \\
\text { clarificado com MgX }\end{array}$ \\
\hline $\begin{array}{c}\text { \% Edulcorantes } \\
\text { \% Compostos das }\end{array}$ & 45,38 & 64,73 & 59,38 \\
$\begin{array}{c}\text { Folhas } \\
\text { \% Redução dos }\end{array}$ & 54,62 & 35,27 & 40,62 \\
$\begin{array}{c}\text { Edulcorantes } \\
\text { \% Redução dos }\end{array}$ & $\# \# \# \#$ & 75,18 & 71,59 \\
Compostos das Folhas & $\# \# \# \#$ & 88,76 & 83,86 \\
\hline
\end{tabular}

Comparando-se as Tabelas 1 e 2, encontram-se porcentagens de edulcorantes e pigmentos distintas para as soluções de caramelo $4 \%$ e $7 \%$, o que certamente não deveria ocorrer. Sabendo-se que a composição do caramelo em pó oscila entre 40 e 50\% em edulcorantes, muito semelhante à composição da solução de ca- 
ramelo $7 \%$ entende-se que, ao se preparar soluções mais diluídas há um aumento na concentração dos edulcorantes, provavelmente devido a uma dissolução mais efetiva dos mesmos. Desta forma, acredita-se que em soluções mais diluídas, durante a preparação das soluções-estoque, os pigmentos sejam mais intensamente retidos na etapa de filtração devido a sua maior concentração na solução original e uma maior solubilidade dos edulcorantes, que são menos retidos.

Já em soluções mais concentradas, como todos os compostos estão em alta concentração, os edulcorantes não modificam sua composição com a solubilização, de modo que a filtração deixa de ser preferencial e não influencia na concentração final da solução.

Os resultados da Tabela 1 mostram que a porcentagem de edulcorantes na solução de caramelo $4 \%$ é mantida praticamente constante, havendo pequeno aumento, variando de $61,41 \%$ para 64,59 e $65,83 \%$ nas soluções clarificadas com CaX e MgX, respectivamente. No caso do caramelo $7 \%$, as porcentagens de edulcorantes das soluções clarificadas são maiores que da solução-estoque, pois aumentam de 45,38 para 64,73 e 59,38\% para as soluções clarificadas com CaX e MgX, respectivamente.

Verifica-se que as soluções clarificadas têm intensidades de dulçor superiores às soluções-estoque, principalmente para soluções mais concentradas. O comportamento das duas soluções sugere que a relação entre edulcorantes e pigmentos das folhas tende a valores da ordem de $65 \%$ de edulcorantes para 35\% de pigmentos, independente da relação inicial, provavelmente devido ao limite de solubilidade dos glicosídeos em solução aquosa na presença dos pigmentos.

Nas duas soluções de caramelo ocorre uma redução global dos constituintes da solução-estoque, de modo que elas adquirem tonalidades bem mais claras, ficando quase límpidas, principalmente no caso das soluções de caramelo 4\%, mas perdem grande massa de edulcorantes que acabam sendo retidos também.

MORAES \& FERNANDES MACHADO [5] realizaram análise de carboidratos totais em chá de Stevia, solução obtida na primeira etapa da extração dos edulcorantes de Stevia rebaudiana, antes e após a adsorção em zeólitas CaX. Os resultados mostraram pequena retenção $(\sim 5 \%)$ dos edulcorantes pelo adsorvente.

Acredita-se que a grande diferença entre estes resultados esteja relacionada à concentração das soluções, aliada à baixa solubilidade dos edulcorantes, pois como o caramelo é um subproduto de processo, constitui um material mais concentrado que o chá, e os compostos com elevada concentração, ao serem retidos pelas zeólitas, acabam arrastando os edulcorantes.

\section{4 - CONCLUSÕES}

Analisando-se os perfis de clarificação apresentados, conclui-se que a zeólita CaX apresenta maior eficácia na purificação da solução de caramelo.

O teste de saturação mostrou que, nas condições de estudo, as zeólitas podem ser reutilizadas por até duas vezes consecutivas como adsorventes na purificação do caramelo sem que ocorra dessorção. Sugerese que, em seguida, seja realizada uma regeneração dos adsorventes para posterior reutilização.

O teste de máxima clarificação produziu soluções quase límpidas, com altos níveis de clarificação (80-90\% dos compostos com maior afinidade), mas com elevadas retenções dos edulcorantes ( $70 \%)$, mostrando que o alto teor de compostos das folhas presentes na suspensão influi na retirada dos edulcorantes da solução já que, quando puros, esteviosídeo e rebaudiosídeo A não são adsorvidos significativamente pela zeólita.

Segundo os resultados de cromatografia líquida de alta eficiência, verificou-se que as zeólitas conseguem reter grande parte dos pigmentos da solução de caramelo, mas arrastam também esteviosídeo e rebaudiosídeo A, fazendo com que o processo apresente baixo rendimento na recuperação dos edulcorantes.

Entretanto, tendo em vista que se trata de um subproduto de processo que vem sendo descartado, o resultado é considerado satisfatório, já que a purificação resulta em um material com sabor e coloração bastante agradáveis, que pode ser aproveitado na elaboração de adoçantes, inclusive melhorando seu dulçor devido à elevada concentração de rebaudiosídeo A no caramelo purificado. Além disso, é importante ressaltar que o processo de adsorção com zeólitas consiste em um tratamento simples e de custo bastante reduzido.

\section{5 - REFERÊNCIAS BIBLIOGRÁFICAS}

[1] GOTO, A. Estudo da Influência do Rebaudiosídeo A na Solubilidade do Esteviosídeo e Análise Sensorial dos Produtos Obtidos. Maringá, 1997. 95p. Dissertação de Mestrado - Departamento de Química, Universidade Estadual de Maringá (UEM).

[2] GOTO, A.; CLEMENTE, E. Influência do Rebaudiosídeo A na Solubilidade e no Sabor do Esteviosídeo. Ciênc. Tecnol. Aliment., v. 18, n. 1, p. 3-6, 1998.

[3] KOTARO, K.; TOKUO, O. Extraction and Purification of Sweetener Component from Dry Leaf of Stevia. Japan Kokai Pat. Appl. 61008020 (Cl. A23L1/22), 1987, 3 p.

[4] MiotTo, D. M. M.; FERNANDES MACHADO, N. R. C. Análise das Alterações Catalíticas em Zeólitas Modificadas utilizadas como Adsorventes. $1^{\circ}$ Congresso Brasileiro de Catálise, v. 2, p. 893-898, 2001.

[5] MiOTTO, D. M. M.; FERNANDES MACHADO, N. R. C. Efeito da Adsorção de Compostos Orgânicos Coloridos sobre Propriedades Ácidas de Zeólitas CaX. V. ERCAT - Encontro Regional de Catálise, p. 146-151, 2002.

[6] MiotTo, D. M. M.; FERnANDES MACHADO, N. R. C. Utilização de Zeólitas Modificadas CaX e MgX como Adsorventes de Compostos Orgânicos. Acta Scientiarum, v. 24, n. 6, p. 1627-1635, 2002.

[7] MORAES, E. P.; FERNANDES MACHADO, N. R. C. Clarification of Stevia rebaudiana (Bert) Bertoni extracted by adsorption in modified zeolites. Acta Scientiarum, v. 23, n. 6, p. 1375-1389, 2001.

\section{6 - AGRADECIMENTOS}

As autoras gostariam de agradecer o suporte financeiro concedido pela Empresa Steviafarma Industrial S.A. 